\title{
Limits on Lorentz violation from the highest energy cosmic rays
}

\author{
Olivier Gagnon and Guy D. Moore \\ Department of Physics, McGill University, \\ 3600 University St., Montréal QC H3A 2T8, Canada
}

(Dated: March 19, 2018)

\begin{abstract}
We place several new limits on Lorentz violating effects, which can modify particles' dispersion relations, by considering the highest energy cosmic rays observed. Since these are hadrons, this involves considering the partonic content of such cosmic rays. We get a number of bounds on differences in maximum propagation speeds, which are typically bounded at the $10^{-21}$ level, and on momentum dependent dispersion corrections of the form $v=1 \pm p^{2} / \Lambda^{2}$, which typically bound $\Lambda>10^{21} \mathrm{GeV}$, well above the Planck scale. For (CPT violating) dispersion correction of the form $v=1+p / \Lambda$, the bounds are up to 15 orders of magnitude beyond the Planck scale.
\end{abstract}




\section{INTRODUCTION}

It is generally believed that Lorentz invariance is an exact symmetry of nature. This belief is supported by extremely precise experimental tests, and by strong and well motivated theoretical prejudice. Indeed, exact Lorentz invariance is used as one of the cornerstones on which relativistic quantum field theory is built, leading to the extremely successful Standard Model of Particle Physics. Together with the equivalence principle, local Lorentz invariance is one of the assumptions underpinning General Relativity. Alternatively, we could say that General Relativity is the gauge theory of general coordinate invariance, and has exact local Lorentz invariance as a consequence. In either case, it is intimately related to both our best theories of particle physics and of gravity.

While most physicists believe that Lorentz symmetry is an exact symmetry of nature, one of our jobs as physicists is to put all of our assumptions to the most vigorous possible tests. Therefore, we should attempt to place the tightest possible limits on the violation of Lorentz symmetry. A general framework for considering Lorentz violation has been developed by Kostelecký and collaborators 1]. The best laboratory experimental limits come from clock comparison experiments, for instance the recent experiments of Cane et. al. [2], see also 3]. These experiments are highly constraining, particularly for low energy effects and those involving CPT symmetry violation. (In quantum field theory, CPT symmetry is a consequence of Lorentz symmetry, together with causality, locality, analyticity, and unitarity, and its violation implies that Lorentz symmetry is also violated [4].)

However, it is consistent and reasonable to believe that CPT could be an exact symmetry without Lorentz invariance. It is also desirable to obtain limits involving rather high energies. In fact, it turns out high energies introduce another way of gaining high precision, in a manner suggested by Coleman and Glashow [5, 6].

The argument goes as follows. If Lorentz symmetry is not exact, then different particles can have different limiting velocities, and potentially more complicated corrections to their dispersion relations. This opens up the possibility of Cherenkov radiation; a particle with a larger limiting velocity is energetically allowed to radiate particles with lower limiting velocities, even though such radiation by a particle at rest is not kinematically allowed. For instance, suppose the limiting velocity of an electron is $c_{e}>c_{\gamma}$, where $c_{\gamma}$ is the limiting velocity of a photon. Then the energy of an electron of momentum $p \gg m c_{e}$ is

$$
E_{e, p}=\sqrt{p^{2} c_{e}^{2}+m_{e}^{2} c_{e}^{4}} \simeq p c_{e}+\frac{m_{e}^{2} c_{e}^{3}}{2 p}
$$

while the energy of a photon of momentum $k$ is $k c_{\gamma}$. The process $e \rightarrow e \gamma$ is kinematically allowed if the final state energies sum to the initial state energy and the sum of the magnitudes of the final state momenta exceeds the magnitude of initial state momentum (so a nonvanishing opening angle is allowed). Denoting the final photon momentum as $k$, defining $\epsilon \equiv\left(c_{e}-c_{\gamma}\right) / c_{\gamma}$, and taking $m_{e}^{2} c_{e}^{2} / p^{2} \sim \epsilon \ll 1$, this condition becomes

$$
\text { Cherenkov condition: } k \epsilon>\frac{m^{2} k c_{e}^{2}}{2 p(p-k)} \text { for some } k, \quad \text { or } \quad \epsilon>\frac{m^{2} c_{e}^{2}}{2 p^{2}} \text {, }
$$

which is the same as the condition that the electron is traveling faster than the speed of light $c_{\gamma}$. This is "traditional" Cherenkov radiation. 
The highest energy particles observed or inferred are not those accelerated in laboratory, but those which occur in cosmic rays or other astrophysical settings. The Cherenkov process is so efficient that an electron which exceeds the threshold for Cherenkov radiation would lose energy down to the threshold on a time scale which is very short compared to any astrophysical time scale. Therefore, electrons of higher energy would never exist astrophysically. One can then place a limit on $\epsilon$ of

$$
\epsilon<\frac{m_{e}^{2} c^{2}}{2 p^{2}},
$$

with $p$ the momentum of the most energetic electron observed or inferred in an astrophysical setting. (Since the constraint on $\epsilon$ will be so tight, there is no need to specify which $c$ appears on the right hand side.)

Similarly, if the photon's limiting velocity is larger, then the process $\gamma \rightarrow e^{+} e^{-}$becomes kinematically allowed for photons of momentum $k$ satisfying

$$
\text { Pair production condition: } k c_{\gamma}>k c_{e}+\frac{2 m_{e}^{2} c^{3}}{k},
$$

where we already used that the process is kinematically most favored when the electron and positron share the momentum evenly. This leads to a bound,

$$
-\epsilon<\frac{2 m_{e}^{2} c^{2}}{k^{2}}
$$

with $k$ the momentum of the most energetic photon observed. The process involved here is not "traditional" Cherenkov radiation, but a sort of "generalized" Cherenkov process.

Observations of cosmic ray photons, and of photons inferred to arise from synchrotron emission off electrons in the Crab nebula, give the constraints 7]

$$
-2 \times 10^{-16}<\epsilon<5 \times 10^{-20}
$$

a two-sided bound on Lorentz violation (though not as strong as those from clock experiments). The authors of [7] also show that such Cherenkov limits place bounds on dispersion corrections involving additional powers of the particle momentum, of form $E=p c+p^{2} / \Lambda$, which limit $\Lambda$ to be at or above the Planck scale (a constraint much stronger than can be obtained from existing laboratory experiments).

One would expect that the strongest limits of this sort would arise from the cosmic rays with the largest energy. The most energetic cosmic rays observed carry energy in excess of $10^{11} \mathrm{GeV}$ and are believed to arise from hadronic primaries [8]. The highest energy cosmic ray observed had an energy of about $3 \times 10^{11} \mathrm{GeV}$ [9], and was probably a hadron [10]. The fact that such high energy particles can arrive from astronomical distances gives the constraints [5, 11],

$$
\begin{gathered}
\frac{c_{p}-c_{\gamma}}{c_{\gamma}}<10^{-23}, \\
\frac{c_{p}-c_{\mathrm{grav}}}{c_{p}}<10^{-15},
\end{gathered}
$$

where $c_{\text {grav }}$ is the propagation speed of gravitation. (The constraint involving gravitation is weaker because gravitational Cherenkov radiation is inefficient; the velocity difference must 
be $10^{-15}$ before gravi-Cherenkov radiation becomes appriciable on an astrophysical time scale.) Unfortunately, these bounds are both one-sided; $c_{p}$ could be substantially slower than $c_{\gamma}$ or $c_{\text {grav }}$ without violating either bound.

The proton is a composite particle. At the energy relevant to the current problem, $10^{11}$ $\mathrm{GeV}$, the proton (as viewed in our rest frame) can be considered as a loose bag of several partons, mostly quarks and gluons, each carrying a small fraction of the proton's total energy. The purpose of this paper is to show that, by taking into account the partonic structure of hadrons, we can use the arrival of high energy hadrons from astronomical distances to place a number of severe limits on Lorentz violating physics. For the reader's convenience, we will present the main results here.

In all of our results, we will assume that the highest energy extensive air showers arise when single hadrons strike the upper atmosphere. (It is possible that heavier nuclei are actually responsible; this would weaken some of our bounds.) For some results, we will make an additional assumption; in a "bottom-up" generation mechanism for the high energy cosmic rays. That is, we will sometimes assume that the highest energy cosmic rays got their energy by being accelerated by ordinary electromagnetic fields over astronomical distances, rather than, for instance, arising as the decay products of some long lived and ultra-heavy relic particles. In this case, the hadron must have been electrically charged during the acceleration process. Therefore, some charged hadron must be stable, at least on the time scale of hours, at the energy of the highest energy cosmic rays observed. In what follows, we will label results which depend on this assumption with an asterisk $*$.

Differences in propagation speeds arise from dimension 4 Lorentz violating operators. We will parameterize these by the propagation speed difference. Choosing to renormalize such operators at the scale $10^{11} \mathrm{GeV}$ (see below), we find the following limits:

$$
\begin{gathered}
-1.6 \times 10^{-23} \quad<\frac{c_{1}-c_{1 / 2}}{c_{1}}<1.4 \times 10^{-23}, \\
* \quad-1.8 \times 10^{-21} \quad<\frac{c_{q}-c_{g}}{c_{g}}<1.2 \times 10^{-21}, \quad * \\
* \quad-2 \times 10^{-21}<\frac{\left(c_{q}+c_{g}\right) / 2-c_{\gamma}}{c_{\gamma}}<5 \times 10^{-24}, \\
* \quad-1 \times 10^{-20}<\frac{\left(c_{q}+c_{g}\right) / 2-c_{e}}{c_{e}}<5 \times 10^{-24} .
\end{gathered}
$$

In the first line, $c_{1}$ and $c_{1 / 2}$ are the propagation speeds of fundamental gauge bosons and fundamental fermions, if propagation speeds are common to particles of a given spin but differ for particles of different spins. In the second line, $c_{q}$ is the propagation speed for quarks assuming all quarks share a maximum velocity, and $c_{g}$ is for gluons. In the remaining lines, $c_{\gamma}$ and $c_{e}$ are the propagation speeds for photons and electrons (the physical particles) respectively; the limits on electrons also apply for neutrinos.

Note that all of these limits are two sided, and many do not require an assumption about the nature of cosmic ray acceleration. These constitute severe constraints on Lorentz violation. In deriving these limits we have assumed that the $3 \times 10^{11} \mathrm{GeV}$ cosmic ray was a hadron and that its energy was correctly measured; the strength of the bound involves the square of the cosmic ray's energy, so if the highest energy cosmic ray hadron energy were only $1 \times 10^{11} \mathrm{GeV}$, the results are an order of magnitude weaker.

Dimension 5 operators induce spin dependent $O\left(p^{2} / \Lambda\right)$ corrections to particle dispersion relations [12], with opposite sign for particle and anti-particle (and for opposite polarization states of gauge fields). There is an independent such parameter for each Standard Model 
field. Note that these operators violate CPT, and so can be avoided by insisting on CPT symmetry.

Unless the coefficient $k$ of these corrections, $E=p \pm k p^{2}$, are at least 20 times larger for QCD degrees of freedom than for photons and leptons, we can obtain the following constraints on $k$ for photons and leptons:

$$
\begin{aligned}
\left|k_{\gamma}\right| & <\frac{1}{10^{34} \mathrm{GeV}}, \\
\left|k_{\nu_{e}}\right|,\left|k_{\nu_{\mu}}\right|,\left|k_{\nu_{\tau}}\right| & <\frac{1}{10^{34} \mathrm{GeV}}, \\
\left|k_{e_{R}}\right|,\left|k_{\mu_{R}}\right| & <\frac{1}{10^{34} \mathrm{GeV}}, \\
\left|k_{\tau_{R}}\right| & <\frac{1}{5 \times 10^{32} \mathrm{GeV}} .
\end{aligned}
$$

These limits are about 15 orders of magnitude stronger than the Planck scale, and do not depend on assumptions about the origins of cosmic rays. This means that any theory which induces dimension 5, CPT violating operators must generate them at a highly transPlanckian scale, or must introduce them with a very small coefficient $\lesssim 10^{-15}$. However, for certain peculiar corners of the parameter space of dimension 5 operators, we cannot place limits. For instance, if the right-handed up quark receives the largest dispersion correction and it is in the range $E=p-k_{u_{R}} p^{2}$, with $k>1 /\left(10^{32} \mathrm{GeV}\right)$; and if dispersion corrections of all non-QCD particles are at least 30 times smaller, then the $\Delta^{++}$may be the most stable propagating particle at the energies of the highest energy cosmic rays, and no limits can be placed.

We are able to place super-Planck scale limits (assuming the Lorentz violating physics introduces the operators with $O(1)$ coefficients) even on dimension 6 operators. Such operators induce CPT-even dispersion corrections of form $E=p+K p^{3}$. The limits we obtain on the coefficient $K$ are,

$$
\begin{aligned}
\frac{-1}{\left(4 \times 10^{22} \mathrm{GeV}\right)^{2}} & <K_{1}<\frac{1}{\left(6 \times 10^{21} \mathrm{GeV}\right)^{2}}, \\
& \frac{-1}{\left(4 \times 10^{22} \mathrm{GeV}\right)^{2}}<K_{1 / 2}<\frac{1}{\left(1.6 \times 10^{22} \mathrm{GeV}\right)^{2}}, \\
* \frac{-1}{\left(6 \times 10^{21} \mathrm{GeV}\right)^{2}} & <K_{q}<\frac{1}{\left(1.6 \times 10^{22} \mathrm{GeV}\right)^{2}}, \\
* & \frac{-1}{\left(2 \times 10^{21} \mathrm{GeV}\right)^{2}}<K_{g}<\frac{1}{\left(6 \times 10^{21} \mathrm{GeV}\right)^{2}}, \\
\frac{-1}{\left(4 \times 10^{22} \mathrm{GeV}\right)^{2}} & <K_{\gamma}<\frac{1}{\left(3 \times 10^{21} \mathrm{GeV}\right)^{2}} \text { or } 25\left|K_{q}\right| * \\
\frac{-1}{\left(4 \times 10^{22} \mathrm{GeV}\right)^{2}} & <K_{\text {lept }}<\frac{1}{\left(1 \times 10^{21} \mathrm{GeV}\right)^{2}} \text { or } 500\left|K_{q}\right| * *
\end{aligned}
$$

Here $K_{1}$ and $K_{1 / 2}$ are the dispersion corrections of spin- 1 and spin- $1 / 2$ particles if these are taken to be common to all particles of the same spin; $K_{q}$ is the dispersion correction of quarks if it is taken to be flavor independent, and $K_{\text {lept }}$ is the largest of the dispersion corrections of any light lepton $\left(e, \mu, \nu_{e}, \nu_{\mu}\right.$, or $\left.\nu_{\tau}\right)$. In the last two lines, the bound is whichever limit is weaker. 
We believe that these constraints will make it next to impossible to develop a theory in which Lorentz symmetry is entirely absent, since they imply a scale of violation at least 2 orders of magnitude higher than the Planck scale. They are rather less constraining if Lorentz symmetry is spontaneously broken in some hidden sector. Note that the scale appearing in the bound arises as $p^{2} / m_{p}$, with $p$ the momentum of the highest energy cosmic ray; the number quoted should be scaled as the second power of that energy, if $3 \times 10^{11} \mathrm{GeV}$ does not hold up as the energy of the highest energy cosmic ray. Some of these bounds, such as the lower bounds on $K_{1}$ and $K_{\gamma}$, apply even if the cosmic rays are nuclei rather than individual hadrons.

The remainder of the paper is organized as follows. In Section [I], we review and discuss what Lorentz violating operators look like and how they generate corrections to dispersion relations. In Section III] we show that the corrections to a hadron's dispersion relation involve the expectation value of the operator in a state given by a highly boosted hadron. This in turn is related to a twist two operator which has a simple expression in terms of the parton distribution functions (PDFs) of the hadron, evaluated at a scale set by the parton's energy in our frame. In Section IV] we present our results for integral moments of parton distribution functions renormalized to the scale $10^{11} \mathrm{GeV}$. Section $\nabla$ shows how the resulting PDFs can lead to specific decay processes which generate the constraints we have just presented. We end with a short conclusion. Finally, details on PDF evolution in the Standard Model are postponed to an appendix.

\section{LORENTZ VIOLATING OPERATORS}

It is fair to assume that any Lorentz violating effects are small, in which case we can treat Lorentz violation as a small perturbation on Lorentz invariant ordinary physics. In the language of field theory, Lorentz violation can be described by the introduction of Lorentz violating operators into the (Standard Model) Lagrangian.

Fairly general discussions of Lorentz violating operators have been presented by Colladay and Kostelecký in a general context, and by Coleman and Glashow in the context of the Standard Model [5]. The most convenient way to introduce such operators is to introduce one or more preferred frame 4-vectors. One then constructs Lagrangian terms as usual, except that Lorentz indices can be contracted against preferred frame 4 -vectors as well as against each other.

Consider for instance electrodynamics with a fermionic field $\psi$ and a gauge boson $A^{\mu}$, with a single timelike 4 -vector $u^{\mu}, u^{\mu} u_{\mu}=-1$, defining a preferred frame ${ }^{1}$. Besides the usual Lagrangian terms,

$$
m \bar{\psi} \psi+\bar{\psi} \gamma^{\mu} D_{\mu} \psi+\frac{1}{4 e^{2}} F_{\mu \nu} F^{\mu \nu}
$$

we can write the new dimension 4 terms,

$$
\left(c_{\psi}^{-1}-1\right) u_{\mu} u_{\nu} \bar{\psi} \gamma^{\mu} D^{\nu} \psi+\frac{c_{\gamma}^{-2}-1}{4 e^{2}} u_{\mu} u^{\nu} F^{\mu \alpha} F_{\nu \alpha},
$$

\footnotetext{
${ }^{1}$ We use $[-+++]$ metric convention.
} 
which modify the free velocities of propagation, in the frame where $u^{\mu}$ is a unit time vector, as indicated. We can eliminate one or the other term by performing a rescaling of the coordinate system, stretching the "time" coordinate (the coordinate which is time in the frame where $u^{\mu}$ points in the time direction), so that one particle velocity again becomes 1. However, the ratio of velocities for the different fields does not change under such a coordinate redefinition, so if this ratio is not 1 , then Lorentz violation cannot be eliminated by any coordinate transformation. ${ }^{2}$

It is also possible to write Lorentz violating operators of both lower and higher dimension. For instance, a dimension 3 term,

$$
u_{\mu} \bar{\psi} \gamma^{\mu} \psi
$$

splits the energies of the particle and antiparticle, a CPT violating effect. At dimension 6, a term of form $-K u_{\mu} u_{\alpha} u_{\beta} u_{\gamma} \bar{\psi} \gamma^{\mu} \partial^{\alpha} \partial^{\beta} \partial^{\gamma} \psi$ modifies the dispersion relation, so that $E-K E^{3}$, rather than $E$, appears (in the frame where $u^{\mu}$ is purely temporal). At high energy and treating the perturbation to be small, the dispersion relation in the rest frame is then approximately $E \simeq p+K p^{3}$, which perturbs the propagation velocity by $v=1+3 K p^{2}$ (setting $c=1)$.

The coefficients of Lorentz violating operators will run with scale, and will generically mix. Rather than investigate this in detail, we will constrain their values at the scale set by the physics which is providing the constraint, which in our case will be $\sim 10^{11} \mathrm{GeV}$.

The absolutely most general Lorentz violating Lagrangian has enough terms to make the analysis very complicated. We will make a number of simplifying assumptions:

1. There is a unique timelike 4 -vector $u^{\mu}$ appearing in Lorentz violating Lagrangian terms, and its preferred frame is not too different from the frame of the microwave background (the boost factor is at most $O(1)$ );

2. For the most part, we will only consider terms even in $u^{\mu}$. Terms odd in $u^{\mu}$ violate CPT, giving opposite corrections to a particle and its CP conjugate.

3. Lorentz violating effects will be family diagonal. We will also present certain constraints assuming they are family blind, or even that they are the same for all gauge bosons and for all fermions.

The last assumption is for convenience, and because we don't know of any strong motivation for theories where it is violated. The other assumptions do not seriously restrict our conclusions, because the cases where they do not hold are typically more strongly constrained. For instance, dropping CPT symmetry allows dimension 3 operators, on which there are very tight laboratory constraints 2]. Nevertheless, we will consider what happens when it is violated-though our ignorance of spin dependent parton distribution functions will make it hard to make as complete an analysis as we make for the CPT symmetric case. Regarding the first assumption, we make two comments. Constraints on spacelike 4-vectors are about

\footnotetext{
${ }^{2}$ It is worth remarking that other considerations, such as causality and locality, may limit what terms can appear in such a Lagrangian [13]. For instance, Kostelecký and Lehnert argue that the term we have written, with $v<1$, cannot be canonically quantized in all frames, while the $v>1$ case may have causality violation issues 13 .
} 
as strong as those on timelike 4-vectors because we have observed ultra-high energy cosmic rays arriving over a wide swath of the sky. For the case where $u^{\mu}$ is purely temporal (or purely spatial) only in a frame of reference at a large boost with respect to our frame, some of the cosmic rays we observe have much higher energies in that frame, and would therefore Cherenkov radiate even more strongly. Therefore such $u^{\mu}$ are more tightly constrained than the $u^{\mu}$ which is at rest in the microwave background frame.

To summarize, Lorentz violating effects behave, at dimension 4, like species dependent speeds of light, and at dimension 6 , like shifts in the propagation speed of form $\delta v \sim \mathbf{p}^{2} / \Lambda^{2}$. Because it is convenient and intuitively clear, we will generally parameterize the Lorentz violating operators in terms of their dispersion relation corrections in the remainder of the paper.

\section{HADRON DISPERSION AND THE TWIST EXPANSION}

How does a Lorentz violating perturbation to the Lagrangian $L_{\mathrm{L}}$ modify a particle's dispersion relation?

First, we must derive from $L_{\mathbb{L}}$ the perturbation to the 4-momentum operator $P_{\square}^{\mu}$. The perturbation to the particle's 4-momentum is then given by

$$
\delta p^{\mu}=\left\langle\psi, \mathbf{p}\left|P_{\amalg}^{\mu}\right| \psi, \mathbf{p}\right\rangle,
$$

where $\psi$ is the particle type and $\mathbf{p}$ is the particle's momentum. The velocity is $v=d p^{0} / d|\mathbf{p}|$, so it is modified, in the ultra-relativistic and small Lorentz violation limit, by $\delta v \simeq d\left(\delta p^{0}-\right.$ $\delta|\mathbf{p}|) / d|\mathbf{p}|$. Since we can shift $L_{\mathbb{L}}$ by a Lorentz conserving piece, we can generally make $\delta|\mathbf{p}|=0$, in which case the correction is just $d \delta p^{0} / d|\mathbf{p}|$. Evaluating this for a free particle is straightforward; we find the part of $L_{\mathbb{L}}$ which looks like a kinetic term for this particle, and replace derivatives with the momentum $p^{\mu}$. The free particle relation between the Lagrangian terms and the modification of particle dispersion relations then holds.

But what if the particle in question is a hadron? Naively, we should view the hadron as a sum of partons with different momentum fractions $x$ of the hadron's momentum, and expect $\delta p^{\mu}$ to be the sum of the contribution expected from each parton. That is, suppose that the dispersion relation for parton type $f$ is

$$
\delta p^{0}[f, \mathbf{p}]=\left\langle f, \mathbf{p}\left|P_{\amalg}^{0}\right| f, \mathbf{p}\right\rangle=\left(c_{f}-1\right)|\mathbf{p}|+K_{f}|\mathbf{p}|^{3} .
$$

Write the parton distribution function for a hadron $h$, such that the probability to find a parton $f$ with momentum fraction between $x$ and $x+d x$ when using a probe of virtuality $q^{2}$ is given by $f_{h}\left(x, q^{2}\right) d x$. We should then expect the correction to the hadron's energy to be,

$$
\delta p^{0}[h, \mathbf{p}]=\left\langle h, \mathbf{p}\left|P_{\amalg}^{0}\right| h, \mathbf{p}\right\rangle=\sum_{f} \int_{0}^{1} d x\left[\left(c_{f}-1\right) x|\mathbf{p}|+K_{f} x^{3}|\mathbf{p}|^{3}\right] f_{h}\left(x,|\mathbf{p}|^{2}\right) .
$$

This basically turns out to be the case.

For our case, $P_{\mathrm{L}}$ is simplest in the rest frame of the 4-vector $u^{\mu}$, and in this frame, $|\mathbf{p}| \gg$ $\Lambda_{\mathrm{QCD}}$, showing that the expectation value of the Lorentz violating operator is determined by hard physics. Alternatively, we could say that $u^{\mu} p_{\mu}$ provides a hard scale which makes 
a partonic description applicable. When evaluating an operator such as $P_{\mathbb{L}}$ in the wave function of a boosted hadron, as in Eq. (3.1), one should perform an operator product expansion on $P_{\mathrm{L}}$ in terms of low twist operators. The dominant contribution will arise from the twist-two part of the operator, with higher twists suppressed by powers of $\Lambda_{\mathrm{QCD}} / u^{\mu} p_{\mu} \sim$ $10^{-11}$.

Consider a twist two, dimension $D$ operator $\mathcal{O}_{D}$. Denote its expectation value in an unphysical state with parton type $f$ carrying momentum $p$ to be

$$
\left\langle f, \mathbf{p}\left|\mathcal{O}_{D}\right| f, \mathbf{p}\right\rangle \equiv\left\langle\mathcal{O}_{D}\right\rangle_{f}
$$

Then the definition of the parton distribution functions (PDFs) is, that the expectation value of $\mathcal{O}_{D}$ in a hadron is given at leading order by

$$
\left\langle h, \mathbf{p}\left|\mathcal{O}_{D}\right| h, \mathbf{p}\right\rangle=\sum_{f}\left\langle\mathcal{O}_{D}\right\rangle_{f} \int_{0}^{1} d x x^{D-3} f_{h}(x)+O\left(\alpha_{\mathrm{s}} / 2 \pi\right),
$$

where $f_{h}(x)$ is the PDF for parton $f$ in the hadron $h$. This is valid up to higher order effects, which are minimized (order $\alpha_{\mathrm{s}} / 2 \pi$ without logarithmic enhancement) if we take the operator $\mathcal{O}$ to be renormalized at the relevant hard scale, which for us is about $10^{11} \mathrm{GeV} .{ }^{3}$ In other words, the expectation values of twist-two operators are given by the Mellin moments of the PDFs-or to be more accurate, at leading order the PDFs are defined as the inverse Mellin transforms of the twist-two moments of the hadron.

Therefore, the correct statement is that the value of an operator in a hadron is the sum over partonic species of the integral over $x$ of the PDF times the expectation of the operator for the given parton. In other words, our naive expectation in Eq. (3.3) is correct, up to $O\left(\alpha_{\mathrm{s}} / 2 \pi\right)$ corrections. Since $\alpha_{s} \sim 0.03$ at the scale in question, we can safely ignore such corrections. (The corrections could be found by making a loop-level treatment of the operator product expansion of the Lorentz violating operator in the twist expansion, and a loop level evaluation of the twist-two operator in terms of the PDFs of the hadron.)

Therefore, the effect of dimension 4 Lorentz violating operators is to give a hadron a limiting velocity of propagation of

$$
c_{h}=\sum_{f} c_{f} \int_{0}^{1} d x x f_{h}(x)
$$

with the sum running over all partonic species. (Note that $\sum_{f} \int_{0}^{1} d x x f_{h}(x)=1$ to ensure momentum conservation.) The limiting velocity of a hadron is the momentum weighted average of the naive limiting velocities of its partonic constituents. Similarly, if parton type

\footnotetext{
3 The Lorentz violating operators are presumably induced at a scale of order the Planck scale and must then be RG evolved from that scale to the scale $10^{11} \mathrm{GeV}$. Such evolution has been studied for the case of QED in 14]. The evolution generically leads to $O(\alpha \log (\Lambda / \mu))$ mixing between operators, making it unlikely that one operator should be smaller than another of the same dimension by more than about a factor of 10. One also expects high dimension operators of dimension $D$ to induce lower dimension ones of dimension $d$ with coefficient $\sim \alpha \Lambda^{D-d}$. This is suggests that dimension 4 operators will have coefficients $O(\alpha)$ [15], which as we will see is grossly in conflict with observation.
} 
$f$ naively gives a dispersion relation of $E=p+K_{f} p^{3}$ due to dimension 6 Lorentz violating operators, then the energy of a hadron will be given by

$$
E=p+p^{3} \sum_{f} K_{f} \int_{0}^{1} d x x^{3} f_{h}(x) .
$$

Since most of the momentum of the hadron is carried at relatively small $x$, this means that the correction to the hadron dispersion, due to dimension 6 operators, will be much smaller than it would be were the hadron not composite.

\section{STANDARD MODEL PARTONIC CONTENT OF HADRONS}

The evolution kernel for parton distribution functions, within QCD, is known to next-toleading order (NLO) 16], and the evolution equations for lower order integral moments are known to NNLO [17, 18]. Global fits of the PDFs of the proton have been performed using data at many energy scales and values of $x[19,20,21]$; those of the neutron are given, to suitable accuracy, by replacing $u \leftrightarrow d$. None of the available PDF libraries go to $10^{11} \mathrm{GeV}$, and none include the evolution of electroweak degrees of freedom (the PDFs of photons and weak bosons, leptons, and the Higgs boson within the proton).

Since some of our most interesting results will come from analyzing the relatively small contamination of electroweak degrees of freedom in hadrons, it behooves us to extend the analysis in this way. Luckily, we are only interested in integral moments of the PDFs, which actually have much simpler evolution equations with scale. Namely, the integral

moments of form $\int_{0}^{1} x^{n} f(x) d x$ obey a closed set of ordinary differential (renormalization group) equations, which can be evolved along with the renormalization group ( $R G$ ) equations for the couplings, once initial values for the integral moments are determined from the global fits. The appendix explains why the integral moments satisfy ordinary differential equations (a point which is well known at least to parts of the community), and extends the usual treatment to include the LO effects of all Standard Model interactions, including electroweak interactions with scalars and Yukawa interactions.

Since we are not generally interested in tracking how much of the structure is in particles and how much in ant-particles, we will only keep track of the sum of the PDFs, $q(x)+\bar{q}(x)$ (the singlet distribution), which we will nevertheless label $q(x)$. We will also not track spin dependent PDFs. The spin dependence and particle-antiparticle difference are both needed to place detailed limits on dimension $5 \mathrm{CPT}$ violating operators, but the spin dependent PDFs are not known with enough accuracy to justify such a treatment anyway.

We have evaluated the relevant integral moments for the quark and gluon PDFs in a proton, neutron, and pion at the relatively low scale of $3 \mathrm{GeV}$, by using a fit from the CTEQ collaboration [19] for the $p, n$ and an old MRST collaboration fit [22] for the pion. These were evolved to the scale $10^{11} \mathrm{GeV}$. In this evolution, gauge field beta functions and QCD contributions to the DGLAP equations (the equations describing the scale dependence of the PDFs) were treated at the two loop level, while the running of the Yukawa coupling and all other DGLAP evolution was treated at one loop level. The Higgs self-coupling was not treated at all, because it first leads to DGLAP evolution at two loops (and then only in the uninteresting Higgs scalar sector) and to Yukawa and gauge boson beta functions 


\begin{tabular}{|c||c|c|c|c|c|c|c|}
\hline Momentum fraction & $p$ & $n$ & $\pi$ & $\gamma$ & $e$ & $D^{0}$ & $g g$ \\
\hline \hline gluon & .454 & .455 & .454 & .019 & .002 & .425 & .491 \\
\hline 1'st gen. quark & .269 & .270 & .266 & .024 & .004 & .122 & .184 \\
\hline 2,3'rd gen. quark & .223 & .223 & .227 & .046 & .008 & .391 & .278 \\
\hline lepton doublet & .002 & .002 & .002 & .054 & .393 & .003 & .002 \\
\hline lepton singlet & .000 & .000 & .000 & .065 & .439 & .001 & .000 \\
\hline U(1) gauge field & .009 & .008 & .008 & .627 & .077 & .013 & .007 \\
\hline SU(2) gauge field & .040 & .040 & .040 & .159 & .075 & .044 & .035 \\
\hline Higgs boson & .003 & .003 & .003 & .007 & .001 & .002 & .003 \\
\hline
\end{tabular}

TABLE I: Dimension 4 content, at $10^{11} \mathrm{GeV}$ scale, of $p, n, \pi, \gamma, e, D^{0}$, and glueball $(g g)$.

at two and three loops respectively. We also treated all thresholds in a rather naive way; for mass thresholds, a particle was excluded from the theory until $q^{2}=m^{2}$, and then included as massless. For the electroweak threshold, taken to occur at $m_{Z}=91.19 \mathrm{GeV}$, we abruptly switched to treating QED+QCD to treating the Standard Model, neglecting all particle masses. At this threshold, the PDF for the $u$ quark was split evenly between the right and left handed components (appropriate for a spin averaged sample of protons), and then the left $u$ type and $d$ type quarks were mixed with each other, while the photonic PDF content was mixed into the $B$ and $W$ boson types according to $\cos ^{2} \Theta_{W}$ and $\sin ^{2} \Theta_{W}$ respectively. (Mixing left quarks would occur anyway due to soft $W$ radiation, a process which is logarithmically divergently efficient.)

This treatment of PDF evolution, as well as the starting PDFs, naturally introduce errors. This is particularly true for the rather poorly known pion PDFs. Note however that the PDF evolution of the integral moments in question tends to converge towards a fixed point, particularly for the strongly interacting degrees of freedom (because of the large size of $\alpha_{\mathrm{s}}$ and the consequent rapid evolution of the PDFs). This is evidenced by the startling similarity of the proton and pion PDFs at the ultraviolet scale. As a consequence, the errors are reduced. In addition, in what follows we will be most interested in the difference between the PDFs of different particles, and the errors in these differences-particularly differences arising because of electroweak interactions-are much smaller. For these reasons, we will not make a detailed error analysis of our PDF evolution. (In any case, our final errors will be dominated by the error in the energy of the highest energy cosmic ray observed to date, which may introduce as much as a factor of 2 error in the numbers in our bounds.) That said, we should particularly point out that the pion PDFs are not as well known, in particular the size of the quark sea is uncertain. Using the extreme large and small sea values given in [22], we find that the gluon fraction in Table \varies by \pm .004 . Fortunately, we do not find that processes involving pions are particularly important in bounding Lorentz violating operators.

We also evolved the PDFs for the electron, the photon, and crude models of the $D$ meson and the glueball. For the electron and the photon, we took the partonic content to be a pure electron or photon with $x=1$ at the scale $500 \mathrm{KeV}$, and evolved under electromagnetic interactions only to the scale $500 \mathrm{MeV}$, and with full interactions from there. This procedure will underestimate the QCD content of the photon somewhat; but we find that neither the photon nor the electron takes on much admixture of other degrees of freedom, and it is not 


\begin{tabular}{|c||c|c|c|c|c|c|c|}
\hline $10^{2} \times \int x^{3} f(x) d x$ & $p$ & $n$ & $\pi$ & $\gamma$ & $e$ & $D^{0}$ & $g g$ \\
\hline \hline gluon & .061 & .062 & .126 & .096 & .004 & .486 & .026 \\
\hline 1'st gen. quark & .427 & .437 & .900 & .699 & .036 & .092 & .043 \\
\hline 2,3'rd gen. quark & .037 & .037 & .076 & 1.35 & .071 & 4.14 & .042 \\
\hline lepton doublet & .001 & .000 & .001 & 2.33 & 30.3 & .006 & .000 \\
\hline lepton singlet & .000 & .000 & .000 & 3.36 & 38.0 & .003 & .000 \\
\hline U(1) gauge field & .007 & .004 & .011 & 62.2 & 1.97 & .083 & .001 \\
\hline SU(2) gauge field & .012 & .012 & .025 & 6.68 & 1.18 & .113 & .002 \\
\hline Higgs boson & .000 & .000 & .001 & .213 & .008 & .002 & .000 \\
\hline Total & .546 & .553 & 1.14 & 76.9 & 71.6 & 4.92 & .114 \\
\hline
\end{tabular}

TABLE II: Dimension 6 content, at $10^{11} \mathrm{GeV}$ scale, of $p, n, \pi, \gamma, e, D^{0}$, and glueball $(g g)$; in percent $(\%)$ of value for a particle with the full $x=1$ fraction of the momentum.

important in practice to use the evolved rather than pure structure.

Treating the $D$ and the glueball requires more modeling. We treat the $D$ meson, at the scale $m_{c} \sim 1.5 \mathrm{GeV}$, as a charm quark with $x=0.8$, with the remaining $20 \%$ of the momentum made up equally of light quark and gluon. The light quark and gluon content are taken to have $\left\langle x^{2}\right\rangle=0.01$ each, so they contribute quite little to the high dimension content of the particle. The structure at higher scales is then obtained by DGLAP running, starting at the scale $1.5 \mathrm{GeV}$. We considered similar models of the $J / \psi$ and $B$ mesons, but these turn out to be too heavy to be important in setting the Lorentz violating bounds. The glueball was taken to be pure glue at the scale $0.5 \mathrm{GeV}$, with $\left\langle x^{2}\right\rangle=0.2$, and was evolved from there. We think this model of the $D^{0}$ is reasonable, but the glueball model is more speculative.

The integral moments we consider can in principle be determined by lattice gauge theory techniques. This would be particularly useful for the glueball, as otherwise we have little guidance in how to treat it.

We find that all QCD particles are about $45 \%$ glue and $50 \%$ quarks, with the remainder made up mostly by gauge bosons. The glueball is richer in glue and the $D$ richer in quarks, but not strikingly so. The photon and electron are about $80 \%$ the original constituent. As for the $x^{2}$ weighted structure, in every case the sum over constituents gives less than 1 (as it must; splitting conserves $\sum x$ but always reduces $\left\langle x^{2}\right\rangle$.) However, for the electron and photon, it is a modest reduction, while for hadrons the reduction is enormous; weighting with $x^{2}$, barely anything is left (or alternately, most of the partonic content has $x<0.1$ ). This is particularly so for the glueball; the root-mean-squared value of $x$ for a glueball constituent is about 0.03 . This is because gluon radiation, which brings down a particle's $x$, is efficient, especially for gluons themselves. 


\section{DERIVATION OF MAIN RESULTS}

\section{A. Dimension 4 operators}

Consider first, constraints on dimension 4 Lorentz violating operators. These look like differences, between particles, in the maximum speed of propagation. Since our constraints will always require the Lorentz violating operators to be subdominant to the Lorentz conserving ones, and since the particles involved will always be highly relativistic, we will always work to linear order in velocity differences, and will not distinguish different particle velocities from each other, and will set $c=1$, after we have made the leading order expansion. Similarly, masses will be taken to correct dispersion relations only at leading order in $\mathrm{m}^{2} / \mathrm{p}^{2}$. So a particle with propagation speed $(1+\epsilon)$ and mass $m$ will be taken to have energy

$$
E=\sqrt{(1+\epsilon)^{2} p^{2}+(1+\epsilon)^{4} m^{2}}=p+\epsilon p+\frac{m^{2}}{2 p} .
$$

Consider first a difference in velocity between fermions and gauge bosons; all fermions have a common propagation velocity at the scale $10^{11} \mathrm{GeV}$, and all gauge bosons have a different common velocity. This could happen if the source of Lorentz violation, whatever it were, distinguished spin but didn't "care" about gauge charges or other internal symmetries.

In this case, it is quite easy to find a two sided bound on the propagation speed difference. Call that difference, $\epsilon \equiv c_{1}-c_{1 / 2}$ (with $c_{1}$ the gauge boson speed and $c_{1 / 2}$ the fermion speed). Consider first the case $\epsilon<0$. In this case, the process $p \rightarrow p \gamma$ is kinematically allowed at suitably high energy. The proton content is almost exactly half each, gauge boson and fermion, whereas the photon is about $80 \%$ gauge boson and only $20 \%$ fermion (see Table I). The energy difference between a proton of momentum $p$, and a final state with collinear proton of momentum $p(1-x)$ and photon of momentum $p x$, is

$$
\begin{aligned}
\Delta E & =(1+0.5 \epsilon) p+\frac{m_{p}^{2}}{2 p}-(1+0.5 \epsilon)(1-x) p-\frac{m_{p}^{2}}{2 p(1-x)}-x(1+0.8 \epsilon) p \\
& =p\left[-0.3 \epsilon x-\frac{x}{1-x} \frac{m^{2}}{2 p^{2}}\right] .
\end{aligned}
$$

When this difference becomes positive, the initial state energy is larger than the final state energy, meaning that the decay can occur with a finite opening angle (which will make the final state energy a bit larger and use up the extra energy). The optimal value of $x$ for this to happen is $x \simeq 1$, and the process becomes kinematically allowed at a threshold,

$$
\epsilon=-\frac{m_{p}^{2}}{0.6 p^{2}}
$$

When this bound is exceeded by a factor of 2 , the proton loses of order half its energy on a length scale of less than a meter; so the bound on cosmic rays can be taken essentially to coincide with the kinematic bound for the process.

Now suppose $\epsilon>0$, so fundamental gauge bosons propagate faster than fundamental fermions. In this case, contrary to simpleminded intuition, the breakup of a proton, $p \rightarrow$ $p e^{+} e^{-}$, becomes allowed, since the electron is about $85 \%$ fermion and only $15 \%$ gauge boson 
(almost exclusively electroweak gauge boson). The kinematics are almost the same as for the previous case, since the electron mass is negligibly small. Combining the two, the bound on $\epsilon$ is,

$$
-\frac{m_{p}^{2}}{0.6 p^{2}}<\left(c_{1}-c_{1 / 2}\right)<\frac{m_{p}^{2}}{0.7 p^{2}} .
$$

Substituting in $m_{p}=0.94 \mathrm{GeV}$ and $p=3 \times 10^{11} \mathrm{GeV}$ gives the bounds quoted in the introduction. Note that neither bound relies on any details of the cosmic ray production mechanism; all we need to know is that the highest energy cosmic rays are hadrons, and that all hadrons are essentially even mixes of gauge boson and fermion.

We have not worried, in the above limit, about scalar (Higgs boson) content, because no low energy particle has much Higgs content. If for some reason $c_{1}$ and $c_{1 / 2}$ were approximately equal and $c_{0}$, the fundamental scalar propagation speed, were more different, we could also achieve limits, about 200 times weaker, by the same processes, because the photon contains more, and the electron less, Higgs boson than any hadron made of light constituents.

These limits can be evaded if we assume that propagation speed differences are not universal but show more complicated species dependence. For instance, suppose that strongly interacting particles propagated slightly more slowly than other degrees of freedom. We can still place a bound, albeit a weaker one, on the propagation speed difference between quarks and gluons, $\epsilon^{\prime} \equiv\left(c_{q}-c_{g}\right)$, as follows.

Suppose first that gluons propagate more slowly. Then a gluon-rich hadron would be energetically more favorable. A hadron of mass $m_{1}$ and gluon fraction $g_{1}$ would split off a daughter hadron of mass $m_{2}$ and gluon fraction $g_{2}$ provided the energy difference,

$$
\begin{aligned}
\Delta E & =\left(1-g_{1} \epsilon^{\prime}\right) p+\frac{m_{1}^{2}}{2 p}-\left(1-g_{1} \epsilon^{\prime}\right)(1-x) p-\frac{m_{1}^{2}}{2 p(1-x)}-\left(1-g_{2} \epsilon^{\prime}\right) x p-\frac{m_{2}^{2}}{2 p x} \\
& =x\left(g_{2}-g_{1}\right) \epsilon^{\prime} p-\frac{m_{1}^{2}}{2 p} \frac{x}{1-x}-\frac{m_{2}^{2}}{2 p x}
\end{aligned}
$$

is positive for any $x$. The most gluon-rich hadron that we have been able to identify is the glueball, a $0^{++}$scalar. There is no conservation rule to forbid the process $p \rightarrow p+g g$ ( $g g$ the glueball). Taking the glueball mass to be $1.6 \mathrm{GeV}$ [23], we find that the process goes forward, for a proton with energy equal to the highest energy cosmic ray observed, if $\epsilon^{\prime}>1.2 \times 10^{-21}$.

The presence of the process $p \rightarrow p+g g$ does not necessarily mean that cosmic rays could not reach the Earth; they would simply have to be composed of glueballs. Even though glueballs are unstable at rest, in the Lorentz violating scenario under discussion, they would be stabilized by the Lorentz violating terms when their energy reached that of the highest energy cosmic rays. Therefore they could propagate to the Earth over cosmological distances. This is provided they were not destroyed by the microwave background; but in fact this seems likely, because a glueball should have a small cross-section with photons. Therefore, this scenario would probably even evade the GZK cutoff [24]. However, we can rule it out if we make the additional assumption that the acceleration mechanism for cosmic rays is by "ordinary" electromagnetic fields. Since the glueball is neutral, it could never achieve more energy than the proton (or other charged hadron) which radiated it, and so the energy limit for the proton is the energy limit for any hadron, within this assumption and assuming as 
well that no other charged hadron would remain stable. This seems safe, as all charged hadrons have valence quarks which give them quark content of order or larger than that of the proton.

In the opposite case, $\epsilon^{\prime}<0$, where gluons propagate faster, a quark-rich hadron is energetically favored. The pion may be, and the kaon is expected to be, slightly quark-richer than the proton, but we find that neither is richer by enough to compete with the process $p \rightarrow p D^{0}$, with the $D^{0}$ a $c \bar{u}$ or $u \bar{c}$ bound state. The kinematic limit for the process, from Eq. (5.5), allows it to occur whenever $\epsilon^{\prime}<-1.8 \times 10^{-21}$. The analogous process with a $D^{ \pm}$requires $6 \mathrm{MeV}$ more energy in the center of mass frame, because of $m_{D^{+}}-m_{D^{0}}$ and $m_{n}-m_{p}$. We have crudely estimated that, when $6 \mathrm{MeV}$ is available in the center of mass frame, the center-of-mass lifetime for the process $p \rightarrow p D^{0}$ is around $10^{-9}$ seconds. The $p \rightarrow p D^{0}$ process occurs before there is enough energy to permit $p \rightarrow n D^{+}$, provided the cosmic ray acceleration takes less than a few hundred seconds (in our frame), which seems reasonable for most proposed cosmic ray sources. Therefore the proton will lose energy to a neutral particle, which will not be accelerated further. The maximum energy a hadron can obtain is therefore the energy of a proton at the kinematic limit for this reaction. This leads to the limits on $\epsilon^{\prime}$ given in the introduction.

We already saw that we can derive a limit when $c_{\gamma}<\left(c_{q}+c_{g}\right) / 2$. We can also set a limit in the opposite case, as follows. The proton has a larger photon (electroweak boson) content than the neutron, and when $c_{\gamma}>c_{q}, c_{g}$, this means the limiting speed of a proton is faster than that of a neutron, opening up the process $p \rightarrow n e^{+} \nu_{e}$ at high enough energies. (The reason the proton has more gauge boson than the neutron is, that it has more up quarks, and the electric charge of the up quark is larger than that of the down quark; so the quarks in a proton radiate more electroweak bosons.) The difference in electroweak boson content, barely discernible in Table 【 is $0.14 \%$ of the proton's momentum. Neglecting for a moment the electron and neutrino masses, this is enough to counter the neutron's larger mass when $\epsilon>.0014 m_{p}\left(m_{n}-m_{p}\right) / p^{2}$. Naively, this leads to a bound of $2 \times 10^{-23}$.

However, this is not the kinematic limit we find, because the timescale for the process $p \rightarrow n e^{+} \nu_{e}$ involves the fifth power of the available energy. Together with the time dilation of the reaction, this leads to a time scale for the reaction, when close to the kinematic limit, of order $10^{14}$ seconds. This is shorter than the likely propagation time of a cosmic ray, but it is much longer than the expected acceleration time of a cosmic ray, which we will take to be $10^{4}$ seconds (of order the time variability of an active galactic nucleus). This knocks two orders of magnitude off our limit. If the correct time scale is shorter, our limit deteriorates as the $1 / 5$ power of the inverse acceleration time. Note also that this limit relies on our assumption of ordinary acceleration. However, the energy involved is enough that the electron mass is negligible, and corrections from different leptonic dispersion relations will be too small to matter, according to constraints we will derive next.

We have already seen how $p \rightarrow p e^{+} e^{-}$gives a strong constraint on the speed difference of $g+q$ and leptons, when the leptons are slower. Similar considerations for $p \rightarrow p \bar{\nu} \nu$ prove that this limit applies both to singlet and doublet neutrinos. However, the limits on the case where the leptons propagate more slowly are much weaker; the analog of the constraint we found for the photon is 15 times weaker, because the lepton content of a hadron is second order in $\alpha$, so the difference between the leptonic content of a neutron and proton is $10^{-4}$. We actually get a stronger bound by combining the two-sided constraints on $c_{\gamma}-\left(c_{q}+c_{g}\right) / 2$, just derived, with the Crab constraints [7] on the electron-photon velocity difference. This 
gives the constraints on the $c_{e}, c_{p}$ differences quoted in the introduction.

The case where different quarks have different limiting velocities is richer, and may be phenomenologically viable; for instance, if the up quark is slower than all other particles, $p \rightarrow \Delta^{++} \pi^{-}$may be allowed, with most of the energy carried by the $\Delta^{++}$. If, on the other hand, the slowest propagating quark is one of the heavier species, say, the charm quark, then $p \rightarrow p D^{0}$ again becomes favored and the proton loses energy to neutral particles; so comparable bounds would exist in this case.

\section{B. Dimension 5 operators}

Next, consider the case with nonvanishing dimension 5 operators. Since they are odd under CPT, these operators always imply opposite dispersion corrections for a particle and its $C P$ conjugate, so if the right-handed ( $\mathrm{SU}(2)$ singlet) up quark has energy $E_{u_{R}}=p+k_{u_{R}} p^{2}$, then the left-handed ( $\mathrm{SU}(2)$ singlet) anti-up has energy $E_{\bar{u}_{R}}=p-k_{u_{R}} p^{2}$. Similarly, opposite polarizations of gauge bosons have opposite corrections. The coefficient $k$ is independent for each distinct Standard Model field; in particular, it is different for right and left handed up quarks, though it is the same for left-handed up and down quarks.

Since we consider this possibility rather unlikely, we will not attempt to be very general in our considerations. Rather, we will assume that the order of magnitude of $k$ is the same for each species, or at least the same within species of a given spin, and try to set some limits on it.

We do not know the spin dependent PDFs of the proton very accurately. What we do know is that the $x^{2}$ moments of PDFs in hadrons are generally quite small, $<0.05$, because most of a hadron's structure at the scale $10^{11} \mathrm{GeV}$ lies in very small $x$ partons. Therefore, if $k$ is comparable for different Standard Model species, then only the dispersion corrections of leptons and electroweak bosons will be important.

With this in mind, consider again the process $p \rightarrow p \gamma$. Take the polarization dependent energy shift for the photon to be $E_{\gamma}=p \pm k_{\gamma} p^{2}$, with \pm according to whether the photon is left or right circularly polarized. The lower energy photon can always be produced; if the helicity of the proton forbids its production, we may consider $p \rightarrow p \gamma \gamma$, with an extra, low energy photon of the opposite polarization. The energy difference for collinear emission, with a photon momentum fraction of $x$, is

$$
\begin{aligned}
\Delta E & =p+\frac{m_{p}^{2}}{2 p}-(1-x) p-\frac{m_{p}^{2}}{2 p(1-x)}-x p+\left|k_{\gamma}\right| x^{2} p^{2} \\
& =-\frac{m_{p}^{2}}{2 p} \frac{x}{1-x}+\left|k_{\gamma}\right| x^{2} p^{2} .
\end{aligned}
$$

This first vanishes when $|k|=2 m_{p}^{2} / p^{3}, x=1 / 2$. Writing $k=1 / \Lambda$, the scale $\Lambda$ is $p^{3} / 2 m_{p}^{2} \simeq$ $1.5 \times 10^{34}$. The result should be slightly less than this, because the structure of the photon is not purely an $x=1$ photon; but looking at Table II we see that not much of the structure has cascaded by this scale, so the correction is quite modest.

We arrive at essentially the same constraint on left-handed leptons by considering $p \rightarrow$ $p \nu \bar{\nu}$; this applies in each generation, since all neutrinos are light. For right handed leptons, the constraints involving electrons are the same, and for muons are weaker only by a factor 
of $2 / 3$, but for $\tau$ leptons they are weaker by a factor of about 20 , due to the mass of the particle to be produced.

In the case where dispersion corrections are more than 30 times larger for QCD degrees of freedom than for leptons or electroweak bosons, these constraints do not apply. New constraints may or may not emerge, depending on exactly what the corrections are. For instance, if $k_{\text {gluon }}$ is much the largest, then $p \rightarrow \Delta g g$, with $g g$ a spin-2 glueball, may be favored. If on the other hand the right handed up quark has the largest $k$, then $p \rightarrow \Delta^{++} \pi^{-}$ will probably occur, and this possibility cannot be excluded on the basis of cosmic rays, since the $\Delta^{++}$is charged and would be the most stable particle at large momenta.

\section{Dimension 6 operators}

If Lorentz symmetry is violated at a high scale, it is difficult to see how to avoid a dispersion correction $E \sim p+O\left(p^{3} / \Lambda^{2}\right)$, with $\Lambda$ a fundamental scale. Presumably $\Lambda \leq m_{\mathrm{pl}}$ the Planck scale, $m_{\mathrm{pl}} \simeq 1.2 \times 10^{19} \mathrm{GeV}$. For instance, if spacetime is a lattice, then lattice dispersion relations of this form are generically generated for free fields. ${ }^{4}$

Simplemindedly, one might expect the most natural form for dimension 6 dispersion corrections to be a universal $K_{1}$ for all gauge fields and a $K_{1 / 2}$ for all fermions. Let us see what limits can be set in this case. If they are of comparable size, then a negative $K_{1}$ leads to photon production, $p \rightarrow p \gamma$, while a negative $K_{1 / 2}$ leads to $p \rightarrow p e^{+} e^{-}$(or neutrinos). Table II shows that only $10 \%$ of a photon's $p^{3}$ weighted content is fermionic, while for the electron less than 5\% is gauge boson; while both particles have over 100 times the size of dimension 6 correction that hadrons have. We therefore neglect the "cross-contamination" of $\gamma$ and $e$, and drop the small dimension 6 content of the hadrons. For the process $p \rightarrow p \gamma$, the process occurs when the energy shift,

$$
\Delta E=\frac{m_{p}^{2}}{2 p} \frac{-x}{1-x}+0.7 K x^{3} p^{3},
$$

is positive. Here 0.7 is $\int x^{3} \gamma(x) d x$ for a photon, from Table III. The resulting bound is, $K_{1}>-1 /\left(4 \times 10^{22} \mathrm{GeV}\right)^{-2}$. For the case $p \rightarrow p e^{+} e^{-}$, we may neglect the electron mass and consider the case where one electron emerges with very small $x$; approximately the same limit is obtained, $K_{1 / 2}>-1 /\left(4 \times 10^{22} \mathrm{GeV}\right)^{-2}$. The scale appearing here is more than 2 orders of magnitude larger than the Planck scale.

For the opposite case, $K_{1}$ or $K_{1 / 2}$ larger than 0 , the proton energy rises faster than linear with $p$ at large momenta, so ordinary Cherenkov radiation of soft photons is allowed, $p \rightarrow p \gamma$, above a threshold of

$$
.0008 K_{1}+.0047 K_{1 / 2}=\frac{m^{2}}{6 p^{4}}
$$

where the numerical constants are the gauge boson and fermion dimension 6 content of the proton from Table II. The numerical values of the bounds are, $K_{1}<\left(6 \times 10^{21} \mathrm{GeV}\right)^{-2}$,

\footnotetext{
${ }^{4}$ Actually, if spacetime is a lattice, then interactions will generically induce $O(\alpha)$ corrections in dimension 4 Lorentz violating operators. We have already seen that such corrections are ruled out by about 20 orders of magnitude.
} 
$K_{1 / 2}<\left(1.6 \times 10^{22} \mathrm{GeV}\right)^{-2}$. Surprisingly, these bounds are only about 4 times weaker; this is due to the more favorable kinematics for the $p \rightarrow p \gamma$ reaction in the positive $K$ scenario.

What if the dimension 6 operators show more species dependence? The cases $K_{\gamma}<0$, $K_{\text {lepton }}<0$ have the same constraints just found; so do the cases $K_{g}>0, K_{q}>0$. For the case $K_{q}<0$ but still universal between flavors, the dominant reaction is $p \rightarrow p D^{0}$, which is allowed by virtue of the larger $x$ quark content of the $D^{0}$ meson. The bound, derived in perfect analogy with the bounds found so far, is $K_{q}>-\left(6 \times 10^{21} \mathrm{GeV}\right)^{-2}$. If $K$ only applies to first generation quarks, the strongest bound is from $p \rightarrow p \pi^{0}$, and the bound is about 3 times weaker (but still exists). A similar bound on $K_{g}$ arises from $p \rightarrow p D^{0}$, $K_{g}>-\left(2 \times 10^{21} \mathrm{GeV}\right)^{-2}$. If we assume $K_{q}, K_{g}=0$, we can also get bounds on positive $K_{\gamma}$, $K_{e}$ from $p \rightarrow p \gamma$ (with $\gamma$ soft), because of the photon and electron content of the proton; these bounds are $K_{\gamma}<\left(3 \times 10^{21} \mathrm{GeV}\right)^{-2}, K_{e}<\left(1 \times 10^{21} \mathrm{GeV}\right)^{-2}$. If $K_{g}, K_{q}$ are nonzero, then the bounds are, $K_{\gamma}<25\left|K_{q}\right|, K_{e}<500\left|K_{q}\right|$ (or the above bound, whichever is weaker). Even these much weaker bounds suggest a trans-Planckian scale.

\section{CONCLUSIONS}

We have shown that the mere existence of hadronic, high energy cosmic rays places constraints on Lorentz violating extensions of the Standard Model which are tighter than any previous constraints for a number of operators. To find these constraints, we first argued that Lorentz violating operators modify particle dispersion relations in a way determined by the partonic content of the particle in question. Then, we determined the partonic content of various hadrons, the photon, and the electron at the relevant scale, set by the energy of the cosmic rays in question. We then looked for Cherenkov-like processes made kinematically possible by these dispersion corrections, and excluded Lorentz violating processes which would prevent extremely high energy, hadronic cosmic rays from existing.

We find that, generally, species dependence in the maximal propagation speed of a particle, induced by dimension 4 Lorentz violating operators, is constrained to be smaller than a part in $10^{21}$. This applied for surprisingly many such dispersion corrections, and all of our limits are two-sided. Limits on dimension 5, CPT violating operators imply that, if generated with $O(1)$ coefficients at the scale of Lorentz symmetry breaking, their intrinsic scale must exceed $10^{34} \mathrm{GeV}$; for dimension 6 operators, the intrinsic scale must exceed $10^{21} \mathrm{GeV}$. It is easy to evade the bounds on dimension 5 operators; they are absent in theories with CPT invariance. The limits on propagation speed differences and dimension 6 operators are harder to avoid in a theory with maximal Lorentz violation in the UV. (Similarly, maximum speed differences in theories without Lorentz symmetry are expected to be $O(\alpha) \sim 10^{-2}$, as recently emphasized by Collins et. al. [15], but it might be possible that there is some loophole in this argument. The argument does not apply if Lorentz symmetry is violated in some hidden sector, in which case the size of Lorentz violating operators in the Standard Model could be much smaller.)

We should finish by commenting on some loopholes and limitations of our analysis. First, the loopholes. Most but not all combinations of Lorentz violating operators can be excluded. In particular, we find the interesting possibility that, if right-handed up quarks have a slower propagation speed than any other particle (either due to dimension 4 corrections or higher order in momentum dependent corrections), then the $\Delta^{++}$particle can be absolutely stable 
at high energies. This possibility may also evade the GZK cutoff. This is an example of the narrow, special corners of the parameter space of Lorentz violating effects which are consistent with observed high energy cosmic ray events.

Also note that not all forms of Lorentz violation lead to dispersion corrections of the form that we have bounded; for instance, spacetime noncommutativity can be understood in terms of the introduction of high dimension, Lorentz violating operators [25], but at

leading order, the operators in question have no twist-two content (see [25]), so they do not modify dispersion relations. We therefore do not obtain strong constraints on spacetime noncommutativity from our results (although this will probably change if we work beyond leading order in the parameter describing the noncommutativity).

One major limitation of our work is that we have considered only the case where there is a single preferred direction; Kostelecký's Standard Model Extension [1] allows more general corrections. Second, we have not been very exhaustive about what sorts of Lorentz violating operators to allow, for instance, generally taking the corrections of all quark flavors to be the same. Third, we have worked within the Standard Model, neglecting the possibility, for instance, that supersymmetry is realized at an energy scale not far above the electroweak one. This latter possibility would require the inclusion of several additional parton distribution functions in our evolution; we would find that the proton and other hadrons have nontrivial gluino and squark content. The tendency of hadronic PDFs to resemble each other would also be strengthened, because $\alpha_{\mathrm{s}}$ would run more slowly and the set of splitting processes available would be larger; therefore limits on, for instance, the quark-gluon velocity difference would be weakened. However, in general we do not believe that this case would be very different from the case of the Standard Model, because we expect the Lorentz violating operators to respect supersymmetry-otherwise, how would we obtain low energy supersymmetry in the first place? We leave these issues to future work.

\section{Acknowledgements}

We would like to thank Michael Luke for useful conversations. This work was supported in part by grants from the Natural Sciences and Engineering Research Council of Canada and by le Fonds Québécois de la Recherche sur la Nature et les Technologies.

\section{APPENDIX A: SPLITTING FUNCTIONS AND COEFFICIENTS IN THE STANDARD MODEL}

The general form of the DGLAP equations is,

$$
\frac{\mu^{2} \partial f_{i}\left(x, \mu^{2}\right)}{\partial \mu^{2}}=\sum_{j} \int_{x}^{1} \frac{d y}{y} P_{i \leftarrow j}(y) f_{j}\left(x / y, \mu^{2}\right) .
$$

Here $f_{i}\left(x, q^{2}\right)$ are the parton distribution functions; $j$ is an index over distinct species. $P_{i \leftarrow j}(y)$ is the rate at which species type $j$ splits to produce species $i$ with momentum fraction $y$. It is generally determined in a series expansion in the coupling, beginning at $O(\alpha / 4 \pi)$. 
The DGLAP splitting functions, at leading order, are determined by the squared matrix element for a particle of momentum $p$ to go to a particle of momentum $x p+p_{\perp}$ and a particle of momentum $(1-x) p-p_{\perp}$, evaluated at leading order in $p_{\perp}^{2}$. Namely, if the matrix element (using the on-shell spinors or perpendicular polarization states as if the particles were on shell) squared, summed on final and averaged over initial spin states, is $\mathcal{M}^{2}$, then the splitting function is given by $\mathcal{M}^{2} p^{3} x(1-x) / 2\left[\mathbf{p} \times\left(x \mathbf{p}+\mathbf{p}_{\perp}\right)\right]^{2}$. That is, the matrix element is to be divided by the squared invariant describing how non-collinear the final states are, and multiplied by the energies of the incoming and outgoing states (when using relativistic state normalization).

For a Lagrangian containing a gauge field and $N_{\mathrm{f}}$ Dirac fermions in representation $f$ under this field,

$$
L=\frac{1}{4 g^{2}} F_{\mu \nu} F^{\mu \nu}+\bar{\psi}(\not D+m) \psi,
$$

we evaluate the splitting function for the process $\psi \rightarrow \psi \gamma$, with $\gamma$ carrying momentum fraction $x$, by evaluating

$$
\left|\bar{u}\left[(1-x) p+p_{\perp}, \sigma_{f}\right] \notin u\left[p, \sigma_{i}\right]\right|^{2},
$$

averaging over initial spins and colors and summing over final spins and colors. Here $u\left[p, \sigma_{i}\right]$ is the spinor for a particle of momentum $p$ and spin $\sigma$. A straightforward and well known calculation leads to the splitting functions,

$$
\begin{aligned}
& P_{\psi \leftarrow \psi}(y)=2 C_{\mathrm{F}}\left(\frac{2}{(1-y)_{+}}-1-y+\frac{3}{2} \delta(1-y)\right), \\
& P_{\psi \leftarrow \gamma}(y)=4 t_{\mathrm{F}} N_{\mathrm{f}}\left(1-2 y+2 y^{2}\right), \\
& P_{\gamma \leftarrow \psi}(y)=2 C_{\mathrm{F}}\left(\frac{2}{y}-2+y\right), \\
& P_{\gamma \leftarrow \gamma}(y)=4 C_{\mathrm{A}}\left(\frac{1}{(1-y)_{+}}+\frac{1}{y}-2+y-y^{2}\right)+\left(\frac{11}{3} C_{\mathrm{A}}-\frac{4}{3} t_{\mathrm{F}} N_{\mathrm{f}}\right) \delta(1-y),
\end{aligned}
$$

each times $\alpha / 4 \pi$. Here $C_{\mathrm{F}}$ is the quadratic Casimir of the fermionic representation $\left(C_{\mathrm{F}}=4 / 3\right.$ for $\mathrm{SU}(3), 3 / 4$ for $\mathrm{SU}(2)$, and $q^{2}$ for $\left.\mathrm{U}(1)\right), C_{\mathrm{A}}$ is the Casimir of the adjoint representation $\left(N_{\mathrm{c}}\right.$ for $\left.\mathrm{SU}\left(N_{\mathrm{c}}\right)\right)$, and $t_{\mathrm{F}}$ is the fermion trace normalization $\left(1 / 2\right.$ for $\mathrm{SU}\left(N_{\mathrm{c}}\right), q^{2}$ for $\left.\mathrm{U}(1)\right)$. Contrary to common usage, the PDF for the fermion is taken in the above to be the sum of the usual fermion and anti-fermion PDF.

If instead we consider a complex scalar field, the quantity to compute is

$$
\left[\left(p+x p+p_{\perp}\right) \cdot \epsilon\right]^{2}
$$

and splitting functions turn out to be $\alpha / 4 \pi$ times

$$
\begin{aligned}
& P_{\phi \leftarrow \phi}(y)=4 C_{\mathrm{S}}\left(\frac{1}{(1-y)_{+}}-1+\delta(1-y)\right), \\
& P_{\phi \leftarrow \gamma}(y)=8 t_{\mathrm{S}} N_{\mathrm{S}}\left(y-y^{2}\right), \\
& P_{\gamma \leftarrow \phi}(y)=4 C_{\mathrm{S}}\left(\frac{1}{y}-1\right), \\
& P_{\gamma \leftarrow \gamma}(y)=[\text { above }]-\frac{2}{3} t_{\mathrm{S}} N_{\mathrm{S}} \delta(1-y) .
\end{aligned}
$$


The DGLAP splitting functions arising from the Lagrangian terms,

$$
L=\left(D_{\mu} H\right)^{\dagger}\left(D^{\mu} H\right)+y_{t}\left(H \bar{t}_{L} P_{R} t_{R}+\text { h.c. }\right),
$$

arise from evaluating $\left|\bar{u}\left(x p+p_{\perp}\right) u(p)\right|^{2}$. For the case where all three fields, $H$, $t_{R}$, and $t_{L}$ are singlets, the splitting functions are $y_{t}^{2} / 16 \pi^{2}$ times

$$
\begin{aligned}
P_{\psi_{i} \leftarrow \psi_{j}}(y) & =(1-y)\left(1-\delta_{i j}\right)-\frac{1}{2} \delta(1-y) \delta_{i j}, \\
P_{\psi_{i} \leftarrow \phi}(y) & =1, \\
P_{\phi \leftarrow \psi_{i}}(y) & =y, \\
P_{\phi \leftarrow \phi}(y) & =-\delta(1-y),
\end{aligned}
$$

where the indices $i, j$ refer to whether the field is $t_{R}$ or $t_{L}$.

The structure of Eq. (A1) means that the integral moments of interest to us obey closed sets of ordinary differential RG equations. This point is "well known to the people who know it well," but may not be familiar to all readers, so we go through it in a little detail. Introduce

$$
f_{i, n}\left(q^{2}\right) \equiv \int_{0}^{1} x^{n} f_{i}\left(x, q^{2}\right) d x
$$

the moment of the PDF corresponding to the expectation value of a dimension $n+3$, twist- 2 operator. We obtain an evolution equation for $f_{i, n}$ from Eq. (A1) by integrating it over $x^{n} d x$;

$$
\begin{aligned}
\int_{0}^{1} x^{n} \frac{q^{2} d f_{i}\left(x, q^{2}\right)}{d q^{2}} d x & =\sum_{j} \int_{0}^{1} x^{n} d x \int_{x}^{1} \frac{d y}{y} P_{i \leftarrow j}(y) f_{j}\left(x / y, q^{2}\right) \\
\frac{q^{2} d f_{i, n}\left(q^{2}\right)}{d q^{2}} & =\sum_{j} \int_{0}^{1} \frac{d y}{y} \int_{0}^{y} x^{n} d x P_{i \leftarrow j}(y) f_{j}\left(x / y, q^{2}\right) \\
& =\sum_{j} \int_{0}^{1} y^{n} d y \int_{0}^{1} z^{n} d z P_{i \leftarrow j}(y) f_{j}\left(z, q^{2}\right) \\
& =\sum_{j}\left(\int_{0}^{1} z^{n} f_{j}\left(z, q^{2}\right) d z\right)\left(\int_{0}^{1} y^{n} d y P_{i \leftarrow j}(y) d y\right) \\
& =\sum_{j} C_{i j, n} f_{j, n}\left(q^{2}\right), \quad \text { with } C_{i j, n} \equiv \int_{0}^{1} y^{n} P_{i \leftarrow j}(y) d y .
\end{aligned}
$$

The evolution equations for the $f_{i, n}$ form a closed set of ordinary differential renormalization group equations, which mix particle species but not operator dimension $n$.

It should not be surprising that the expectation values of all dimension $D$, twist 2 operators should satisfy closed renormalization group equations in this way. We expect an operator to mix under renormalization group flow only with operators of the same spin and equal or lower dimension. Since twist 2 operators have the maximum spin available at a given dimension, the twist 2 operators of dimension $D$ can only mix with other twist 2 operators at dimension $D$.

It remains to evaluate the $C_{i j}$ for all particles of the Standard Model, from the splitting functions we have just given. This is a straightforward combinatorial problem. We will write 
the fermionic fields as $[E, L, U, D, Q]$ for the right-handed electron, leptonic doublet, righthanded up, right-handed down, and quark doublet fields, $[B, W, G]$ for the hypercharge, weak SU(2), and strong SU(3) (gluon) gauge bosons, and $H$ for the Higgs doublet. The couplings are $\left[a^{\prime}, a, a_{s}, a_{y}\right]$, with $a=\alpha / 4 \pi$ and $a_{y}=y^{2} / 16 \pi^{2}$ with $y$ the Yukawa coupling of the top quark, $m_{t}^{2}=y^{2} v^{2} / 2$ ( $v$ the Higgs vacuum expectation value, $\left.G_{F}=1 / v^{2} \sqrt{2}\right)$. The evolution equations for the integral moments of the PDFs are, at leading order,

$$
\begin{aligned}
& \frac{q^{2} d}{d q^{2}} E_{m}=a^{\prime} C_{f f} E_{m}+a^{\prime} C_{f g} B \\
& \frac{q^{2} d}{d q^{2}} L_{m}=\left(\frac{1}{4} a^{\prime}+\frac{3}{4} a\right) C_{f f} L_{m}+\frac{1}{2} a C_{f g} W+\frac{1}{2} a^{\prime} C_{f g} B, \\
& \frac{q^{2} d}{d q^{2}} D_{m}=\left(\frac{4}{3} a_{s}+\frac{1}{9} a^{\prime}\right) C_{f f} D_{m}+\frac{1}{2} C_{f g} a_{s} G+\frac{1}{3} C_{f g} a^{\prime} B \text {, } \\
& \frac{q^{2} d}{d q^{2}} U_{m}=\left(\frac{4}{3} a_{s}+\frac{4}{9} a^{\prime}\right) C_{f f} U_{m}+\frac{1}{2} C_{f g} a_{s} G+\frac{4}{3} C_{f g} a^{\prime} B \\
& +a_{y} \delta_{m 3}\left[2 C_{f f y} U_{3}+C_{f 2 f y} Q_{3}+C_{f s} H\right] \text {, } \\
& \frac{q^{2} d}{d q^{2}} Q_{m}=\left(\frac{4}{3} a_{s}+\frac{3}{4} a+\frac{1}{36} a^{\prime}\right) C_{f f} Q_{m}+\frac{1}{2} C_{f g} a_{s} G+\frac{3}{2} C_{f g} a W+\frac{1}{6} C_{f g} a^{\prime} B \\
& +a_{y} \delta_{m 3}\left[C_{f f y} Q_{3}+2 C_{f 2 f y} U_{3}+C_{f s} H\right] \text {, } \\
& \frac{q^{2} d}{d q^{2}} B=a^{\prime} C_{g f} \sum_{m}\left(E_{m}+\frac{1}{4} L_{m}+\frac{1}{9} D_{m}+\frac{4}{9} U_{m}+\frac{1}{36} Q_{m}\right)+\frac{10 N_{\mathrm{g}}}{3} a^{\prime} C_{g g f} B \\
& +\frac{1}{4} a^{\prime} C_{g s} H+\frac{1}{2} a^{\prime} C_{g g s} B \text {, } \\
& \frac{q^{2} d}{d q^{2}} W=a C_{g f} \sum_{m}\left(\frac{3}{4} L_{m}+\frac{3}{4} Q_{m}\right)+2 N_{\mathrm{g}} a C_{g g f} W+2 a C_{g g g} W \\
& +\frac{3}{4} a C_{g s} H+\frac{1}{2} a C_{g g s} W \text {, } \\
& \frac{q^{2} d}{d q^{2}} G=a_{s} C_{g f} \sum_{m}\left(\frac{4}{3} U_{m}+\frac{4}{3} D_{m}+\frac{4}{3} Q_{m}\right)+2 N_{\mathrm{g}} a_{s} C_{g g f} G+3 a_{s} C_{g g g} G, \\
& \frac{q^{2} d}{d q^{2}} H=\left(\frac{3}{4} a+\frac{1}{4} a^{\prime}\right) C_{s s g} H+\frac{1}{2} a C_{s g} W+\frac{1}{2} a^{\prime} C_{s g} B \\
& +a_{y}\left[C_{s s y} H+C_{s f}\left(Q_{3}+2 U_{3}\right)\right] \text {, }
\end{aligned}
$$

where $m$ is a generation index, $N_{g}=3$ is the number of generations, and the $C_{x x x}$ coefficients are integrals of splitting functions given explicitly by,

$$
\begin{aligned}
& C_{f f}=\frac{-8}{3}, \quad C_{g f}=\frac{8}{3}, \quad C_{f g}=\frac{2}{3}, \quad C_{g g f}=\frac{-2}{3}, \quad C_{g g g}=0, \\
& C_{s s g}=-2, \quad C_{g s}=2, \quad C_{s g}=\frac{1}{3}, \quad C_{g g s}=\frac{-1}{3}, \\
& C_{f f y}=\frac{-1}{2}, \quad C_{f 2 f y}=\frac{1}{6}, \quad C_{s f}=\frac{1}{3}, \quad C_{f s}=\frac{1}{2}, \quad C_{s s y}=-1,
\end{aligned}
$$


for the case of the dimension 4 integral moments and

$$
\begin{aligned}
& C_{f f}=\frac{-157}{30}, \quad C_{g f}=\frac{11}{15}, \quad C_{f g}=\frac{11}{30}, \quad C_{g g f}=\frac{-2}{3}, \quad C_{g g g}=\frac{-21}{5}, \\
& C_{s s g}=\frac{-13}{3}, \quad C_{g s}=\frac{1}{3}, \quad C_{s g}=\frac{2}{15}, \quad C_{g g s}=\frac{-1}{3}, \\
& C_{f f y}=\frac{-1}{2}, \quad C_{f 2 f y}=\frac{1}{20}, \quad C_{s f}=\frac{1}{5}, \quad C_{f s}=\frac{1}{4}, \quad C_{s s y}=-1,
\end{aligned}
$$

for the case of dimension 6 integral moments. The $a_{s}^{2}$ corrections can be found in [18], and the Standard Model beta functions are in [26], and we will not repeat them here.

[1] See for instance, D. Colladay and V. A. Kostelecký, Phys. Rev. D 58, 116002 (1998) hep-ph/9809521.

[2] F. Cane et al., physics/0309070, D. F. Phillips, M. A. Humphrey, E. M. Mattison, R. E. Stoner, R. F. C. Vessot and R. L. Walsworth, Phys. Rev. D 63, 111101 (2001) physics/0008230.

[3] S. K. Lamoreaux, J. P. Jacobs, B. R. Heckel, F. J. Raab and E. N. Fortson, Phys. Rev. Lett. 57, 3125 (1986); C. D. Lane, PhD. thesis, Indiana University, 2000 (UMI-99-93635); R. Bluhm, V. A. Kostelecký, C. D. Lane and N. Russell, Phys. Rev. Lett. 88, 090801 (2002) hep-ph/0111141 ; R. L. Walsworth, D. Bear, M. Humphrey, E. M. Mattison, D. F. Phillips, R. E. Stoner and R. F. C. Vessot, AIP Conf. Proc. 539, 119 (2000) physics/0007063; R. L. Walsworth and D. F. Phillips, physics/0007062; V. A. Kostelecký and C. D. Lane, Phys. Rev. D 60, 116010 (1999) hep-ph/9908504.

[4] O. W. Greenberg, Phys. Rev. Lett. 89, 231602 (2002) hep-ph/0201258.

[5] S. R. Coleman and S. L. Glashow, Phys. Rev. D 59, 116008 (1999) hep-ph/9812418.

[6] S. R. Coleman and S. L. Glashow, hep-ph/9808446 Phys. Lett. B 405, 249 (1997) hep-ph/9703240.

[7] T. A. Jacobson, S. Liberati, D. Mattingly and F. W. Stecker, astro-ph/0309681; T. Jacobson, S. Liberati and D. Mattingly, Nature 424, 1019 (2003) astro-ph/0212190; Phys. Rev. D 66, 081302 (2002) hep-ph/0112207.

[8] M. Nagano and A. A. Watson, Rev. Mod. Phys. 72, 689 (2000).

[9] D. J. Bird et al., Astrophys. J. 441, 144 (1995).

[10] M. Risse, P. Homola, D. Gora, J. Pekala, B. Wilczynska and H. Wilczynski, astro-ph/0401629

[11] G. D. Moore and A. E. Nelson, JHEP 0109, 023 (2001) hep-ph/0106220.

[12] R. C. Myers and M. Pospelov, Phys. Rev. Lett. 90, 211601 (2003) hep-ph/0301124.

[13] V. A. Kostelecký and R. Lehnert, Phys. Rev. D 63, 065008 (2001) arXiv:hep-th/0012060.

[14] V. A. Kostelecký, C. D. Lane and A. G. M. Pickering, Phys. Rev. D 65, 056006 (2002) arXiv:hep-th/0111123.

[15] J. Collins, A. Perez, D. Sudarsky, L. Urrutia and H. Vucetich, gr-qc/0403053

[16] G. Curci, W. Furmanski and R. Petronzio, Nucl. Phys. B 175, 27 (1980).

[17] A. Retey and J. A. M. Vermaseren, Nucl. Phys. B 604, 281 (2001) hep-ph/0007294.

[18] S. A. Larin, P. Nogueira, T. van Ritbergen and J. A. M. Vermaseren, Nucl. Phys. B 492, 338 (1997) hep-ph/9605317. 
[19] J. Pumplin, D. R. Stump, J. Huston, H. L. Lai, P. Nadolsky and W. K. Tung, JHEP 0207, 012 (2002) hep-ph/0201195, see also http://www.phys.psu.edu/ cteq/\#PDFs

[20] A. D. Martin, R. G. Roberts, W. J. Stirling and R. S. Thorne, hep-ph/0307262 Phys. Lett. B 531, 216 (2002) hep-ph/0201127. See also, http://durpdg.dur.ac.uk/HEPDATA/PDF.

[21] M. Gluck, E. Reya and A. Vogt, Eur. Phys. J. C 5, 461 (1998) hep-ph/9806404.

[22] P. J. Sutton, A. D. Martin, R. G. Roberts and W. J. Stirling, Phys. Rev. D 45, 2349 (1992).

[23] C. J. Morningstar and M. J. Peardon, Phys. Rev. D 60, 034509 (1999) hep-lat/9901004.

[24] K. Greisen, Phys. Rev. Lett. 16, 748 (1966); G. T. Zatsepin and V. A. Kuzmin, JETP Lett. 4, 78 (1966) [Pisma Zh. Eksp. Teor. Fiz. 4, 114 (1966)].

[25] S. M. Carroll, J. A. Harvey, V. A. Kostelecký, C. D. Lane and T. Okamoto, Phys. Rev. Lett. 87, 141601 (2001) hep-th/0105082.

[26] C. Ford, D. R. T. Jones, P. W. Stephenson and M. B. Einhorn, Nucl. Phys. B 395, 17 (1993) hep-lat/9210033. 